\title{
Fetal Position
}

National Cancer Institute

\section{Source}

National Cancer Institute. Fetal Position. NCI Thesaurus. Code C92926.

The position of the fetus in the uterus. 